\title{
Selection of piperacillin/tazobactam infusion mode guided by SOFA score in cancer patients with hospital-acquired pneumonia: a randomized controlled study
}

This article was published in the following Dove Press journal:

Therapeutics and Clinical Risk Management

\begin{abstract}
Yang Lyu, ,,* Yang Yang, ${ }^{2, *}$ Xin $\mathrm{Li}^{3}$ Min Peng, ${ }^{3}$ Xin $\mathrm{He},{ }^{2}$ Peng Zhang, ${ }^{4}$ Shangwen Dong, ${ }^{4}$ Wanhua Wang, ${ }^{2}$ Donghao Wang'

'Department of Intensive Care Unit, Tianjin Medical University Cancer Institute and Hospital, National Clinical Research Center for Cancer, Key Laboratory of Cancer Prevention and Therapy, Tianjin, Tianjin's Clinical Research Center for Cancer, ${ }^{2}$ Department of Anesthesia, Tianjin Medical University Cancer Institute and Hospital, National Clinical Research Center for Cancer, Key Laboratory of Cancer Prevention and Therapy, Tianjin, Tianjin's Clinical Research Center for Cancer, ${ }^{3}$ Department of Intensive Care Unit, Tianjin Medical University General Hospital, Tianjin, ${ }^{4}$ Department of Cardiothoracic Surgery, Tianjin Medical University General Hospital, Tianjin, China

*These authors contributed equally to this work
\end{abstract}

Correspondence: Donghao Wang Department of Intensive Care Unit, Tianjin Medical University Cancer Institute and Hospital, National Clinical Research Center for Cancer, Key Laboratory of Cancer Prevention and Therapy, Tianjin, Tianjin's Clinical Research Center for Cancer, Huan-Hu-Xi Road, Ti-Yuan-Bei, He Xi District, Tianjin 300060, China

Tel +862223340I23

Email yang_lu@tmu.edu.cn

\begin{abstract}
Background: This study aimed to select piperacillin/tazobactam (TZP) infusion mode guided by Sequential Organ Failure Assessment (SOFA) score in cancer patients with hospital-acquired pneumonia (HAP) postoperation.

Patients and methods: A total of 120 cancer patients with postoperative HAP were divided into two groups: improved administration group (L group) and conventional treatment group (Con group). The Con group received traditional infusion of TZP and the L group received it as prolonged infusion. Blood drug concentration was detected at different time points. Based on the SOFA cut-off value of 9, the patients were regrouped into M (mild) and S (severe) groups.
\end{abstract}

Results: Percent time that the free drug concentrations remain above the minimum inhibitory concentration (\%fT $>$ MIC) was longer than $5 \mathrm{~h}$ in L group, but $<4 \mathrm{~h}$ in Con group. Administration method ( $p=0.033$, OX value $2.796, B$ value $1.028,95 \% \mathrm{CI}: 0.855-8.934$ ) and SOFA score ( $p=0.038$, OX value $0.080, B$ value $-2.522,95 \%$ CI: $0.007-0.874$ ) were independent predictors of patient survival. In the $\mathrm{S}$ group, compared to conventional treatment, prolonged infusion mode resulted in shorter days of antibiotic use and shorter ventilator time, and achieved longer survival, better clinical efficacy, and lower 28-day mortality rate.

Conclusion: For cancer patients with SOFA score $\geq 9$, prolonged infusion of TZP could benefit the patients and obtain better clinical efficacy.

Keywords: cancer, Sequential Organ Failure Assessment score, piperacillin, tazobactam, pneumonia, antibiotics

\section{Introduction}

With the increase in the variety of widely used antibiotics, drug-resistant strains emerge as the pathogens of hospital-acquired pneumonia (HAP), including resistant Gramnegative bacteria such as Pseudomonas aeruginosa, Acinetobacter spp., Enterobacter cloacae, Klebsiella pneumoniae, and Escherichia coli and Gram-positive methicillinresistant Staphylococcus aureus. ${ }^{1}$ Maximizing the antibacterial activity of antibiotics and choosing correct, effective, safe, and economical administration of antibiotics are extremely urgent and necessary.

Percent time that the free drug concentration remains above the minimum inhibitory concentration (MIC), that is, \%fT $>\mathrm{MIC}$, is an important index of the pharmacodynamics of $\beta$-lactam antibiotics. Piperacillin/tazobactam (TZP) administration aims to extend \% $\mathrm{fT}>\mathrm{MIC}$ to improve clinical outcomes. In general, we can increase the dose and the frequency of administration, or extend the infusion time to increase the 
efficacy of the drug. However, increasing the dose and the frequency of administration would increase treatment cost and resource consumption. Therefore, we chose extending infusion time to increase the antibacterial activity of TZP and improve the clinical efficacy without increasing the dose of TZP. The severity of the patient's condition could determine the clinical efficacy. Therefore, we introduced the Sequential Organ Failure Assessment (SOFA) score to assess the patient's condition prior to administration. We hypothesized that the SOFA score could guide us in choosing the correct administration to maximize the antimicrobial activity of TZP.

\section{Patients and methods}

\section{Patient selection}

A randomized, prospective, controlled study, approved by the Medical Ethics Committee of Tianjin Medical University Cancer Hospital with approval number E2011077A, was conducted. Written informed consent was obtained from all patients or their families. The clinical trial registration was completed and approved with registration certificate number NCT01796717 (https://register.clinicaltrials.gov).

The inclusion criteria were as follows: age 18-70 years; cancer and postoperative HAP diagnosed in accordance with the Chinese Medical Association, American College of Chest Physicians, and the Society of Infectious Disease diagnostic criteria; ${ }^{2,3}$ no cardiopulmonary disease before operations; no lung injury and respiratory dysfunction during surgery and anesthesia; no pulmonary metastasis before operations; abdominal tumor patients who had received radical resection; no bleeding, fistula, abdominal infection, and cardiac dysfunction postoperation; and the pathogens should be sensitive to TZP, with MIC of 8 or $16 \mathrm{mg} / \mathrm{L}$.

The exclusion criteria were as follows: clinical manifestations of infection and having received systematic anti-infection treatment $>48 \mathrm{~h}$ before enrolling; sepsis with hypotension and/or evidence of organ dysfunction (septic shock): systolic blood pressure $<90 \mathrm{mmHg}$ or diastolic blood pressure $<60 \mathrm{mmHg}$, receiving vasoactive drugs $>4 \mathrm{~h}$; kidney injury: urine output $<20 \mathrm{~mL} / \mathrm{h}$ or $<80 \mathrm{~mL} / 4 \mathrm{~h}$, acute renal failure, requiring blood purification treatment, creatinine clearance $<20 \mathrm{~mL} / \mathrm{min}$; infections caused by bacteria not susceptible to TZP; recurrent lung infections caused by complicated factors such as obstructive pneumonia, lung abscess, empyema, and active tuberculosis; allergic to penicillin; pregnant or lactating women; any predictable factors which may increase the risk for patients or interfere with the results of clinical trials in patients, such as anastomotic fistula after abdominal tumor operation, acute liver failure, acute cardiac and cerebral vascular accident.

\section{Treatment}

Before treatment, all patients were assessed with SOFA score. In accordance with the sputum culture and susceptibility results, TZP (trade name for Tazocin) was selected for antiinfection treatment. All patients were randomly divided into the following two groups by a computer, to generate random numbers without prior restrictions or intervention or adjustment in advance or during the implementation process: improved administration group (L group, 60 cases) and conventional treatment group (Con group, 60 cases). In accordance with the "National Guidelines for Antimicrobial Therapy" issued by the Medical Administration Department of the Ministry of Health in the People's Republic of China, Con group received TZP at $4.5 \mathrm{~g}$ per $6 \mathrm{~h}$ and intravenous infusion was completed within $30 \mathrm{~min}$; the $\mathrm{L}$ group received TZP at $4.5 \mathrm{~g}$ per $6 \mathrm{~h}$ with an infusion pump to extend the administration time to $3 \mathrm{~h}$. The nurses in charge were blind to the grouping.

\section{Determination of serum drug concentration}

Two millilitres of peripheral blood was collected from all enrolled patients at $0.5,1,2,3,4,5$, and $6 \mathrm{~h}$ after drug administration in a heparin tube and centrifuged for $10 \mathrm{~min}$, immediately. The plasma was then separated and the specimen placed at $-80^{\circ} \mathrm{C}$.

Ultra-performance liquid chromatography tandem mass spectrometry was used to determine TZP concentration in the plasma at the Tianjin Medical University Cancer Hospital Pharmacological Laboratory.

\section{Clinical therapeutic efficacy}

Before and after 3 days of treatment, the two groups were compared for acute physiology and chronic health evaluation II (APACHE II) score, clinical pulmonary infection score (CPIS), and procalcitonin (PCT) level. The clinical efficacy and bacteriologic efficacy were determined in accordance with the "Guiding principles for clinical application of antimicrobial agents" issued by the General Logistics Department of the Ministry of Health in the People's Republic of China. The successful treatment rate of the two groups was assessed, and SOFA sensitivity and specificity were calculated for the cut-off value of SOFA score. Based on the SOFA cut-off value, patients were regrouped into mild group 
(M group) and severe group (S group). Based on different modes of administration of TZP, the patients in each group were divided into extending administration group (including M1 and S1 groups) and conventional treatment group (including M2 and S2 groups).

\section{Statistical analysis}

SPSS 19.0 statistical software was applied for data processing. Data with normal distribution were presented as mean \pm SD and compared using independent $t$-test. Chi-square test or Fisher's exact probability method was used for counting data. Cox or logistic regression was used for multivariate analysis. $p<0.05$ was considered statistically significant.

\section{Results}

\section{Comparison of the patients in $L$ and Con groups}

In this study, we enrolled 120 patients, including 30 cases of lung cancer (25 cases of squamous cell carcinoma of the lung and five cases of lung adenocarcinoma), 56 cases of upper gastrointestinal cancer (21 cases of gastric cancer, 15 cases of hepatocellular carcinoma, six cases of cholangiocarcinoma, and 14 cases of pancreatic cancer), 30 cases of lower gastrointestinal cancer (21 cases of colon cancer and nine cases of rectal cancer), and four cases of renal carcinoma.

With regard to HAP, 29 cases had $P$. aeruginosa, 18 cases had Es. coli, 22 cases had K. pneumoniae, 21 cases had Acinetobacter baumannii, eight cases had Viscous serratia, and 19 cases had En. cloacae infections. Regarding incision classification, 30 cases had chest incision, 59 cases had abdominal incision, and 31 cases had thoracoabdominal incision. There were no significant differences in general data of the patients in $\mathrm{L}$ and Con groups before treatment (Tables 1 and 2).

However, after administration of TZP, \%fT $>$ MIC time was $>5 \mathrm{~h}$ in L group and $<4 \mathrm{~h}$ in Con group, with MIC of 8 or $16 \mathrm{mg} / \mathrm{L}$ (Figure 1 ).

\section{Sensitivity and specificity of SOFA score for treatment outcome and prognosis}

Cox regression analysis showed that administration method ( $p=0.033$, OX value $2.796, B$ value $1.028,95 \% \mathrm{CI}$ : $0.855-8.934)$ and SOFA score $(p=0.038$, OX value 0.080 , $B$ value -2.522 , 95\% CI: $0.007-0.874)$ were independent predictors of patient survival. APACHE II score $(p=0.745$, $B$ value 0.015$)$, CPIS score $(p=0.743, B$ value -0.049$)$, oxygenation index (mmHg; $p=0.212, B$ value 0.012 ), PCT (ng/mL; $p=0.655, B$ value 0.003$)$, albumin $(\mathrm{g} / \mathrm{L} ; p=0.127$, $B$ value -0.118$)$, primary disease classification $(p=0.655$, $B$ value 9.066$)$, incision classification $(p=0.820, B$ value 0.162 ), and initial pathogen classification ( $p=0.720, B$ value 7.237) had no significant effect on patient survival.

Receiver operating characteristic curve analysis showed that the areas under the curve of SOFA score, APACHE II, and CPIS score were $0.726 \pm 0.050,0.685 \pm 0.060$, and $0.453 \pm 0.062$, respectively. SOFA score had the highest area under the curve value. When the SOFA score was 9 , the sensitivity was $84 \%$ and the specificity was $57 \%$. Thus, we set the cut-off value of SOFA as 9. This value had high sensitivity and specificity to distinguish mild and severe cases and for selection of different antibiotics (Figure 2).

Table I The comparison of clinical data before treatment

\begin{tabular}{|c|c|c|c|c|c|c|c|c|c|}
\hline & \multicolumn{3}{|c|}{$L$ and Con groups $(n=\mid 20)$} & \multicolumn{3}{|c|}{ M group $(n=53)$} & \multicolumn{3}{|c|}{ S group $(n=67)$} \\
\hline & L group & Con group & $p$-value & MI group & M2 group & $p$-value & SI group & S2 group & $p$-value \\
\hline & $(n=60)$ & $(n=60)$ & & $(n=29)$ & $(n=24)$ & & $(n=32)$ & $(n=35)$ & \\
\hline SOFA score & $10.77 \pm 5.896$ & $11.85 \pm 5.719$ & 0.309 & $5.45 \pm 1.502$ & $5.58 \pm 1.442$ & 0.741 & $15.56 \pm 3.732$ & $16.20 \pm 2.837$ & 0.432 \\
\hline APACHE II score & $20.35 \pm 5.272$ & $22.68 \pm 7.736$ & 0.056 & $16.83 \pm 3.496$ & $16.04 \pm 3.099$ & 0.395 & $25.25 \pm 4.197$ & $27.17 \pm 6.883$ & 0.177 \\
\hline CPIS score & $8.85 \pm 1.793$ & $8.53 \pm 1.546$ & 0.302 & $8.55 \pm 1.429$ & $8.21 \pm 1.318$ & 0.368 & $9.19 \pm 2.055$ & $8.69 \pm 1.641$ & 0.277 \\
\hline MIC (mg/L) & $11.07 \pm 3.922$ & II $.47 \pm 3.998$ & 0.581 & $11.03 \pm 3.950$ & $11.00 \pm 3.956$ & 0.975 & $11.25 \pm 3.992$ & $11.66 \pm 4.043$ & 0.680 \\
\hline Ol (mmHg) & $204.42 \pm 34.36$ & $198.28 \pm 34.03$ & 0.328 & $227.7 \pm 25.19$ & $225.5 \pm 24.15$ & 0.746 & $|82.3 \pm 26.3|$ & $180.2 \pm 27.26$ & 0.751 \\
\hline PCT (ng/mL) & $6.26 \pm 6.950$ & $5.98 \pm 6.058$ & 0.815 & $5.66 \pm 5.367$ & $5.56 \pm 7.856$ & 0.957 & $6.21 \pm 5.893$ & $6.60 \pm 6.665$ & 0.799 \\
\hline Albumin (g/L) & $26.25 \pm 2.972$ & $26.10 \pm 2.874$ & 0.779 & $26.34 \pm 2.349$ & $27.42 \pm 2.083$ & 0.088 & $26.16 \pm 3.122$ & $25.54 \pm 3.257$ & 0.435 \\
\hline $\mathrm{Ccr}(\mathrm{mL} / \mathrm{min})$ & $61.40 \pm 6.730$ & $63.43 \pm 7.335$ & 0.116 & $66.38 \pm 6.758$ & $65.29 \pm 6.511$ & 0.556 & $61.25 \pm 7.535$ & $60.34 \pm 6.183$ & 0.591 \\
\hline
\end{tabular}

Notes: The Con group (conventional treatment group) received traditional infusion of TZP and the L group (improved administration group) received it as prolonged infusion. Blood drug concentration was detected at different time points. Based on the SOFA cut-off value of 9 , the patients were regrouped into $M$ (mild) and $S$ (severe) groups. MI: mild group with prolonged administration; M2: mild group with traditional administration; SI: severe group with prolonged administration; S2: severe group with traditional administration.

Abbreviations: APACHE II, acute physiology and chronic health evaluation II; Ccr, creatinine clearance; CPIS, clinical pulmonary infection score; MIC, minimum inhibitory concentration; OI, oxygenation index; PCT, procalcitonin; SOFA, Sequential Organ Failure Assessment. 
Table 2 The comparison of general data before treatment

\begin{tabular}{|c|c|c|c|c|c|c|c|c|c|}
\hline & \multicolumn{3}{|c|}{$L$ and Con groups $(n=\mid 20)$} & \multicolumn{3}{|c|}{$M$ group $(n=53)$} & \multicolumn{3}{|c|}{$S$ group $(n=67)$} \\
\hline & L group & Con group & $p$-value & MI group & M2 group & p-value & SI group & S2 group & $p$-value \\
\hline & $(n=60)$ & $(n=60)$ & & $(n=29)$ & $(n=24)$ & & $(n=32)$ & $(n=35)$ & \\
\hline Age (years) & $67.40 \pm 7.727$ & $69.50 \pm 6.604$ & 0.112 & $67.34 \pm 7.898$ & $69.9 \pm 6.171$ & 0.200 & $67.5 \pm 7.573$ & $69.2 \pm 7.051$ & 0.337 \\
\hline Gender (male/female) & $37 / 23$ & $29 / 31$ & 0.144 & $17 / 12$ & $11 / 13$ & 0.358 & $20 / 12$ & $18 / 17$ & 0.365 \\
\hline BMI $\left(\mathrm{kg} / \mathrm{m}^{2}\right)$ & $22.37 \pm 1.946$ & $23.29 \pm 4.033$ & 0.109 & $22.23 \pm 1.868$ & $23.25 \pm 3.802$ & 0.210 & $22.45 \pm 2.012$ & $23.36 \pm 4.285$ & 0.273 \\
\hline Primary disease (cases) & & & 0.332 & & & 0.710 & & & 0.877 \\
\hline Lung cancer & 14 & 16 & & 9 & 4 & & 8 & 8 & \\
\hline UGC & 26 & 30 & & 11 & 13 & & 15 & 16 & \\
\hline LGC & 18 & 12 & & 6 & 7 & & 7 & II & \\
\hline Other cancers & 2 & 2 & & 3 & 0 & & 2 & 0 & \\
\hline Incision site (cases) & & & 0.698 & & & 0.175 & & & 0.869 \\
\hline Chest incision & 14 & 16 & & 9 & 4 & & 8 & 8 & \\
\hline Abdominal incision & 33 & 26 & & 14 & 12 & & 16 & 18 & \\
\hline $\mathrm{TI}$ & 13 & 18 & & 6 & 8 & & 8 & 9 & \\
\hline Initial pathogen (cases) & & & 0.860 & & & 0.407 & & & 0.833 \\
\hline PA & 14 & 15 & & 10 & 9 & & 6 & 6 & \\
\hline EC & 7 & II & & 4 & 4 & & 3 & 8 & \\
\hline $\mathrm{KP}$ & 15 & 7 & & 4 & 4 & & 8 & 6 & \\
\hline$A B$ & 12 & 9 & & I & 4 & & 9 & 5 & \\
\hline SM & 2 & 6 & & 2 & 3 & & I & 4 & \\
\hline $\mathrm{EC}$ & 9 & 10 & & 6 & 0 & & 4 & 6 & \\
\hline Other & $\mathrm{f}^{\mathrm{a}}$ & $2^{\mathrm{b}}$ & & 2 & 0 & & I & 0 & \\
\hline
\end{tabular}

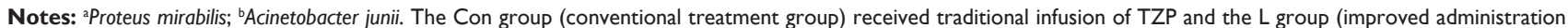
group) received it as prolonged infusion. Blood drug concentration was detected at different time points. Based on the SOFA cut-off value of 9 , the patients were regrouped into $\mathrm{M}$ (mild) and S (severe) groups. MI: mild group with prolonged administration; M2: mild group with traditional administration; SI: severe group with prolonged administration; S2: severe group with traditional administration.

Abbreviations: AB, Acinetobacter baumannii; BMI, body mass index; EC, Enterobacter cloacae; EC, Escherichia coli; KP, Klebsiella pneumoniae; LGC, lower gastrointestinal cancer; PA, Pseudomonas aeruginosa; SM, Serratia marcescens; TI, thoracoabdominal incision; UGC, upper gastrointestinal cancer.

\section{Comparison of patients in $\mathrm{M}$ and $\mathrm{S}$ groups}

According to the cut-off value of SOFA, the patients were divided into mild disease group (M group, SOFA $<9$ ) and severe group ( $\mathrm{S}$ group, $\mathrm{SOFA} \geq 9$ ). There were 53 cases in M group, including 29 cases in the prolonged administration group (M1) and 24 cases in the traditional administration group (M2). There were 67 cases in S group, including 32 cases in the prolonged administration group (S1) and 35 cases in the traditional administration group (S2). We compared the basic data of the four groups of patients before and after treatment.

First, we compared the clinical data between L and Con groups after treatment and found that prolonged infusion time of administration improved patient survival, increased clinical efficacy and bacteriologic efficacy, and reduced 28-day mortality $(p<0.05)$, as shown in Table 3 and Figure 3.

Next, in the two mild groups M1 and M2, bacteriologic efficacy was significantly different. However, the clinical
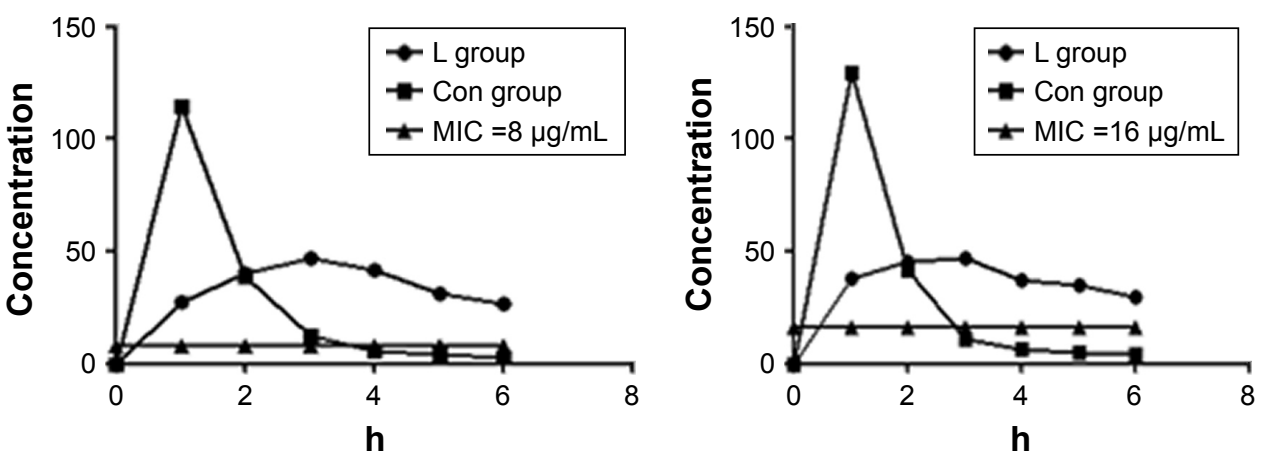

Figure I \% T $>$ MIC in L group and Con group with MIC of 8 and $16 \mu g / \mathrm{mL}$.

Notes: The Con group (conventional treatment group) received traditional infusion of TZP and the L group (improved administration group) received it as prolonged infusion. $\% \mathrm{fT}>\mathrm{MIC}$ represents the percent time that free drug concentrations remain above the MIC.

Abbreviation: MIC, minimum inhibitory concentration. 


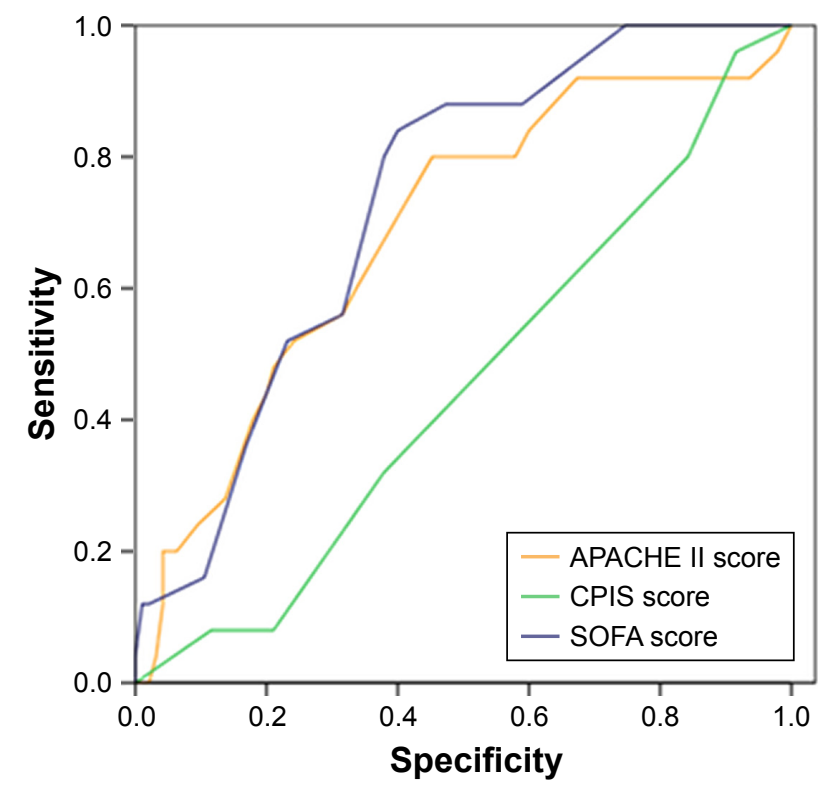

Figure 2 ROC curve of SOFA score, APACHE II and CPIS score. When the SOFA score was 9 , the sensitivity was $84 \%$ and the specificity was $57 \%$.

Abbreviations: APACHE II, acute physiology and chronic health evaluation II; CPIS, clinical pulmonary infection score; ROC, receiver operating characteristic; SOFA, Sequential Organ Failure Assessment.

indicators of survival, days of antibiotic use, ventilator time, clinical efficacy, and 28-day mortality in the two groups showed no significant difference $(p>0.05$; Table 3$)$. There was no significant difference in survival between the M1 and M2 groups (Figure 4).

Furthermore, in the two severe groups S1 and S2, bacteriologic efficacy was significantly different. In addition, days of antibiotics use and ventilator time were shorter in $\mathrm{S} 1$ group than in $\mathrm{S} 2$ group. The patients in S1 group had longer survival, better clinical efficacy, and lower 28-day mortality rate than those in S2 group. The survival of $\mathrm{S} 1$ group was superior to that of S2 group (Figure 5).

\section{Discussion}

Blindly choosing the highest level of antibiotics may produce good effect in the short term, but in the long run, the consequences arising from drug resistance of bacteria may be disastrous. ${ }^{4}$ TZP is a mixed preparation of broadspectrum $\beta$-lactam antibiotic piperacillin and $\beta$-lactamase inhibitor tazobactam. The $\% \mathrm{fT}>\mathrm{MIC}$ index is a measure of bactericidal effect. ${ }^{5}$ Increasing the dosage or the frequency of administration can show increased efficacy, but increases the medical costs and causes varying degrees of kidney damage. Prolonging the infusion time may be a desirable dosing regimen to increase drug efficacy without increasing its dosage. ${ }^{6}$ Our previous study showed that TZP-optimized dosing regimen of prolonging the infusion time improved clinical efficacy, shortened treatment time, and reduced medical cost. ${ }^{7}$ Critically ill patients are the population most likely to benefit from the optimization strategy of drug exposure. ${ }^{8,9}$ Lodise et al found that in patients with $P$. aeruginosa infection and APACHE II score $\geq 17$, extending the TZP infusion time significantly reduced mortality and the number of days of hospitalization, compared to the traditional mode of administration. ${ }^{10}$ A recent study reported that continuous infusion of TZP achieved similar efficacy and safety to conventional intermittent infusion. ${ }^{11}$ However, a large multicenter study confirmed that prolonged infusion led to significantly better clinical cure and survival rates compared with intermittent infusion. ${ }^{12}$

In this study, we divided all patients into two groups randomly, to receive TZP in different modes of administration. We detected the plasma concentration of TZP at different time points and found that at MIC of 8 or $16 \mathrm{mg} / \mathrm{L}$, the $\% \mathrm{fT}>\mathrm{MIC}$ was longer than $5 \mathrm{~h}$ in L group but $<4 \mathrm{~h}$ in the control group. Statistical analysis showed that survival

Table 3 The comparison of clinical data in each group after treatment

\begin{tabular}{|c|c|c|c|c|c|c|c|c|c|}
\hline & \multicolumn{3}{|c|}{$\underline{L}$ and Con groups $(n=\mid 20)$} & \multicolumn{3}{|c|}{ M group $(n=53)$} & \multicolumn{3}{|c|}{$S$ group $(n=67)$} \\
\hline & L group & Con group & $p$-value & MI group & M2 group & p-value & SI group & S2 group & $p$-value \\
\hline & $(n=60)$ & $(n=60)$ & & $(n=29)$ & $(n=24)$ & & $(n=32)$ & $(n=35)$ & \\
\hline Antibiotic usage (days) & $\mathrm{II} .12 \mathrm{I} \pm 0.772$ & $13.227 \pm 0.605$ & 0.124 & $11.48 \pm 3.55$ & $10.42 \pm 2.569$ & 0.225 & $12.94 \pm 3.340$ & $14.66 \pm 3.386$ & $0.04 I$ \\
\hline Ventilator time $(\mathrm{h})$ & $|45.95 \pm 2| .46$ & $152.01 \pm 35.78$ & 0.515 & $5.79 \pm 4.15$ & $6.13 \pm 4.28$ & 0.956 & $126.09 \pm 12.91$ & $169.36 \pm 16.45$ & 0.043 \\
\hline Survival time (days) & $27.77 \pm 0.127$ & $24.15 \pm 1.029$ & 0.027 & $28.00 \pm 0.00$ & $27.17 \pm 4.082$ & 0.276 & $27.56 \pm 1.243$ & $21.69 \pm 9.048$ & 0.001 \\
\hline Clinical efficacy (\%) & 88.33 & 86.67 & 0.044 & 96.55 & 96.55 & 0.448 & 78.13 & 57.14 & 0.007 \\
\hline BE (\%) & 98.33 & 75.00 & 0.000 & 100.00 & 83.33 & 0.011 & 96.88 & 71.43 & 0.005 \\
\hline 28-day mortality (\%) & 1.67 & 8.33 & 0.023 & 0.00 & 0.00 & 1.000 & 3.12 & 14.29 & 0.027 \\
\hline
\end{tabular}

Notes: The Con group (conventional treatment group) received traditional infusion of TZP and the L group (improved administration group) received it as prolonged infusion. Blood drug concentration was detected at different time points. Based on the SOFA cut-off value of 9 , the patients were regrouped into $M$ (mild) and S (severe) groups. MI: mild group with prolonged administration; M2: mild group with traditional administration; SI: severe group with prolonged administration; S2: severe group with traditional administration.

Abbreviation: BE, bacteriologic efficacy. 


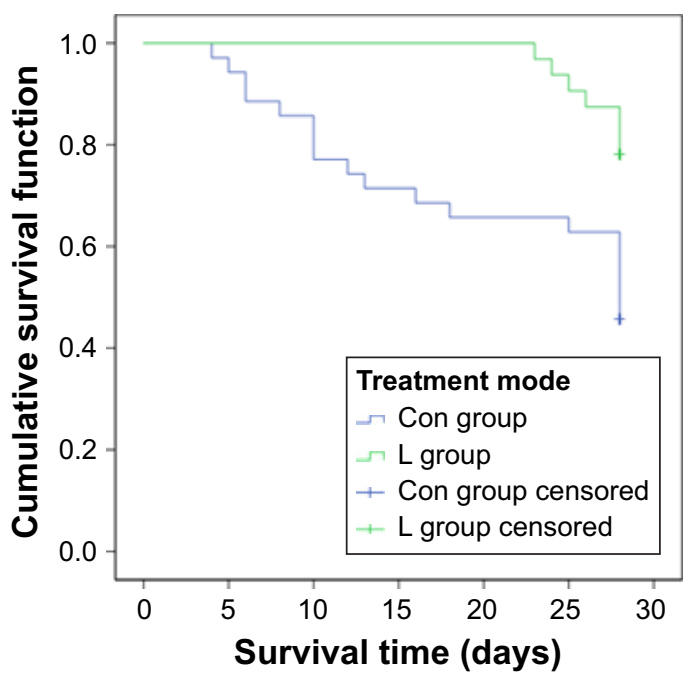

Figure 3 Survival curve of $L$ group and Con group.

Note: The Con group (conventional treatment group) received traditional infusion of TZP and the L group (improved administration group) received it as prolonged infusion.

was better in L group than in control group, and prolonged infusion increased the clinical efficacy and bacteriologic efficacy while reducing the mortality. However, in clinical practice, we found that some patients do not need prolonged infusion of TZP, but still show good outcome. Prolonged infusion mode depends on the use of infusion pumps, which increases the cost of treatment, and it may also increase the risk of venous catheter infection.

Therefore, it is important to identify patients who need prolonged infusion mode the most. In this study, we used Cox

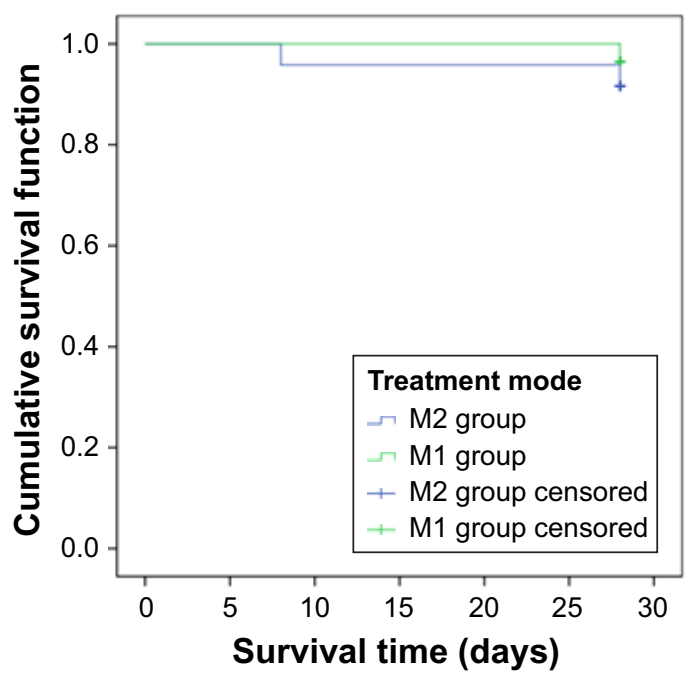

Figure 4 Survival curve of MI and M2 groups.

Notes: There was no significant difference in the survival time between MI and M2 groups. The Con group (conventional treatment group) received traditional infusion of TZP and the L group (improved administration group) received it as prolonged infusion. Blood drug concentration was detected at different time points. Based on the SOFA cut-off value of 9 , the patients were regrouped into $M$ (mild) and $\mathrm{S}$ (severe) groups. MI: mild group with prolonged administration; M2: mild group with traditional administration.

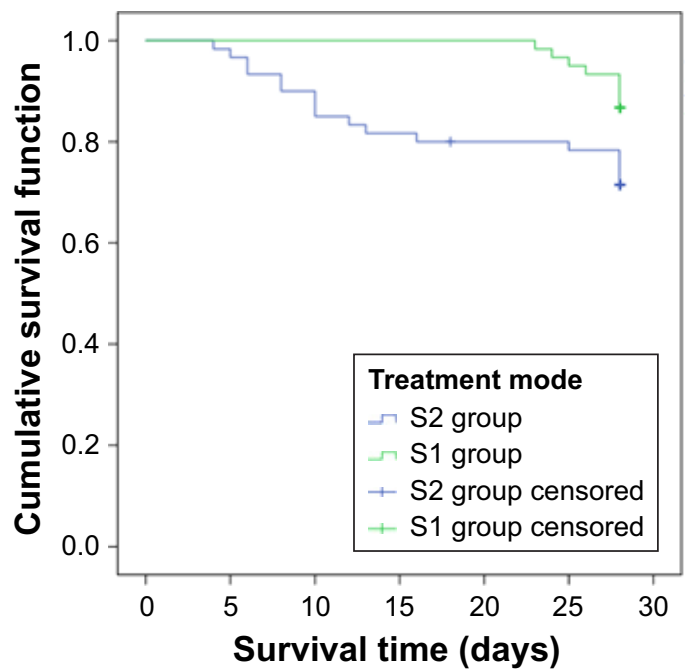

Figure 5 Survival curve of SI and S2 groups.

Notes: The survival of S1 group was better than that of S2 group. The Con group (conventional treatment group) received traditional infusion of TZP and the $L$ group (improved administration group) received it as prolonged infusion. Blood drug concentration was detected at different time points. Based on the SOFA cutoff value of 9 , the patients were regrouped into $M$ (mild) and $S$ (severe) groups. S1: severe group with prolonged administration; S2: severe group with traditional administration.

regression analysis to compare the effects of various factors on the survival of patients and found that administration method ( $p=0.033$, OX value $2.796, B$ value $1.028,95 \% \mathrm{CI}$ : $0.855-8.934)$ and SOFA scores $(p=0.038$, OX value 0.080 , $B$ value -2.522 , 95\% CI: $0.007-0.874)$ were independent predictors of survival. SOFA score has been proposed by the European Association of Intensive Care Medicine; it is simple, objective, easily accessible, and noninvasive for the assessment of critically ill patients, and increased scores indicate more severe diseases. ${ }^{13}$

We recorded APACHE II score, CPIS score, PCT, treatment effect, and prognosis after the treatment. We detected the sensitivity and specificity of the SOFA score before treatment to assess the prognosis for patients and found that a SOFA cut-off value of 9 may have higher sensitivity and specificity. All patients were regrouped in accordance with this cut-off value and divided into M1, M2, S1, and S2 groups in accordance with the different modes of administration of TZP and severity of the disease.

In the mild groups $\mathrm{M} 1$ and $\mathrm{M} 2$ receiving different modes of TZP administration, the days of antibiotics use, ventilator time, clinical and bacteriologic efficacy, and even 28-day mortality and survival time had almost no significant differences between groups. However, in the severe groups S1 and S2, we found significant differences in bacteriologic efficacy, and the days of antibiotic use and the ventilator time were shorter in S1 group than in S2 group after treatment. In addition, the survival was longer, clinical efficacy 
was better, and 28-day mortality was lower in S1 group than in S2 group.

Therefore, for nonsevere HAP patients, we can obtain similar clinical efficacy using extended or traditional administration methods for antibiotics. However, for severe HAP patients, the selection of TZP prolonged infusion for the treatment of infection by resistant bacteria $(\mathrm{MIC}=8 / 16 \mathrm{mg} / \mathrm{L})$ may highlight the advantages of \%fT $>$ MIC. The antibacterial activity can be maximized by choosing prolonged infusion of TZP.

Several limitations of this study should be stated. First, the sample size of this study is relatively small. Large-scale multicenter studies with more than 500 subjects are needed to confirm our conclusion. Second, we could not exclude some confounding factors that interfere with the correlation of SOFA value with different modes of administration, which may cause bias in our conclusion. Such confounding factors include organ dysfunction, especially kidney dysfunction, and circulation and respiration status. Therefore, it is important to include subjects with the same or similar status of organ function for comparison.

In conclusion, compared to the traditional infusion method of TZP, prolonged infusion exhibits better efficacy. However, not all patients can benefit from prolonged infusion. For severe patients with SOFA score $\geq 9$, prolonged infusion of TZP could show significantly better clinical efficacy.

\section{Acknowledgments}

The study was supported by a grant from the Science and Technology fund of Tianjin Municipal Health Bureau, China (No 2013KZ091) and the Science Foundation of Tianjin Medical University, China (No 2014KYQ10).

\section{Disclosure}

The authors report no conflicts of interest in this work.

\section{References}

1. Nseir S, Grailles G, Soury-Lavergne A, Minacori F, Alves I, Durocher A. Accuracy of American Thoracic Society/Infectious Diseases Society of America criteria in predicting infection or colonization with multidrugresistant bacteria at intensive-care unit admission. Clin Microbiol Infect. 2010;16(7):902-908.

2. Chinese Society of Respiratory Disease Association. Hospital-acquired pneumonia diagnosis and treatment guidelines. Modern Practical Medicine. 2002;14:160-161.

3. American thoracic society; Infectious Diseases Society of America. Guidelines for the management of adults with hospital-acquired, ventilator-associated and healthcare-associated pneumonia. Am J Respir Crit Med. 2005;171(4):388-416.

4. Li J, Song X, Yang T, et al. A systematic review of antibiotic prescription associated with upper respiratory tract infections in China. Medicine (Baltimore). 2016;95(19):e3587.

5. Zhou YF, Shi W, et al. Pharmacokinetic/pharmacodynamic correlation of cefquinome against experimental catheter-associated biofilm infection due to Staphylococcus aureus. Front Microbiol. 2016;6:1513.

6. Lee LS, Kiinzig-Schippers M, Nafziger AN, et al. Comparison of $30 \mathrm{~min}$ and $3 \mathrm{~h}$ infusion regimens for imipenem/cilastatin and meropenem evaluated by Monte Carlo Simulation. Diagn Microbiol Infect Dis. 2010;68(3):251-258.

7. Lü Y, Yan Z, Wang DH, Dong WL, Yang Y, Xia R. [Treatment study of hospital acquired pneumonia by optimizing dosing regimen of piperacillin/tazobactam:prolonged versus. regular infusion]. Zhonghua Wei Zhong Bing Ji Jiu Yi Xue. 2013;25(8):479-483. Chinese.

8. Langan KM, Jacob J, Li J, et al. Pharmacokinetics of short versus extended infusion meropenem dosing in critically ill patients: a pilot study. Crit Care Resusc. 2014;16(3):190-196.

9. Lee GC, Liou H, Yee R, Quan CF, Neldner K. Outcomes of extendednfusion piperacillin-tazobactam: a retrospective analysis of critically ill patients. Clin Ther. 2012;34(12):2297-2300.

10. Lodise TP Jr, Lomaestro B, Drusano GL. Piperacillin-tazobactam for Pseudomonas aeruginosa infection: clinical implications of an extendedinfusion dosing strategy. Clin Infect Dis. 2007;44(3):357-363.

11. Cotrina-Luque J, Gil-Navarro MV, Acosta-García H, et al. Continuous versus intermittent piperacillin/tazobactam infusion in infection due to or suspected pseudomonas aeruginosa. Int J Clin Pharm. 2016; 38(1):70-79.

12. Abdul-Aziz MH, Lipman J, Akova M, et al; DALI Study Group. Is prolonged infusion of piperacillin/tazobactam and meropenem in critically ill patients associated with improved pharmacokinetic/ pharmacodynamic and patient outcomes? An observation from the Defining Antibiotic Levels in Intensive care unit patients (DALI) cohort. J Antimicrob Chemother. 2016;71(1):196-207.

13. Peres Bota D, Melot C, Lopes Ferreira F, Nguyen Ba V, Vincent JL. The multiple organ dysfunction score (MODS) versus the sequential organ failure assessment (SOFA) score in outcome prediction. Intensive Care Med. 2002;28(11):1619-1624.
Therapeutics and Clinical Risk Management

\section{Publish your work in this journal}

Therapeutics and Clinical Risk Management is an international, peerreviewed journal of clinical therapeutics and risk management, focusing on concise rapid reporting of clinical studies in all therapeutic areas outcomes, safety, and programs for the effective, safe, and sustained use of medicines. This journal is indexed on PubMed Central, CAS,

\section{Dovepress}

EMBase, Scopus and the Elsevier Bibliographic databases. The manuscript management system is completely online and includes a very quick and fair peer-review system, which is all easy to use. Visit $\mathrm{http}: / / \mathrm{www}$.dovepress.com/testimonials.php to read real quotes from published authors. 